\title{
Fiber Fabry-Perot interferometer sensor for measuring resonances of piezoelectric elements
}

Ricardo E. da Silva, Roberson A. Oliveira, Alexandre A. P. Pohl

Ricardo E. da Silva, Roberson A. Oliveira, Alexandre A. P. Pohl, "Fiber FabryPerot interferometer sensor for measuring resonances of piezoelectric elements," Proc. SPIE 7753, 21st International Conference on Optical Fiber Sensors, 775319 (17 May 2011); doi: 10.1117/12.885858

SPIE Event: 21st International Conference on Optical Fibre Sensors (OFS21), 2011, Ottawa, Canada 


\title{
Fiber Fabry-Perot interferometer sensor for measuring resonances of piezoelectric elements
}

\author{
Ricardo E. da Silva, Roberson A. Oliveira, Alexandre A. P. Pohl \\ Federal University of Technology-Paraná, 3165 Av. Sete de Setembro, Curitiba,Brazil, 80.230-90 \\ Graduate School of Electrical Engineering and Computer Science
}

\begin{abstract}
The development of a fiber extrinsic Fabry-Perot interferometer for measuring vibration amplitude and resonances of piezoelectric elements is reported. The signal demodulation method based on the use of an optical spectrum analyzer allows the measurement of displacements and resonances with high resolution. The technique consists basically in monitoring changes in the intensity or the wavelength of a single interferometric fringe at a point of high sensitivity in the sensor response curve. For sensor calibration, three signal processing techniques were employed. Vibration amplitude measurement with $0.84 \mathrm{~nm} / \mathrm{V}$ sensitivity and the characterization of the piezo resonance is demonstrated.
\end{abstract}

Keywords: Fabry-Perot interferometer, optical signal processing, piezoelectrics

\section{INTRODUCTION}

Extrinsic Fabry-Perot interferometer (EFPI) has received much attention in the last years due to advantages such as high resolution, electromagnetic immunity and large multiplexing capability. The device is basically composed of a cavity formed by the cleaved interface of an optical fiber and the sample which serves as a mirror. The relative displacement of the sample may be converted into phase, polarization, frequency, wavelength modulation or a combination of any of these. For measuring vibration amplitudes and resonances of zirconate titanate-lead (PZT) elements using photodetectors and oscilloscopes, a simple fringe-counting technique can be employed. But fluctuation and noise in coherent sources impact the resolution and response time of conventional arrangements, limiting its application to low frequencies, which very often requires advanced signal processing techniques ${ }^{1}$. The use of EFPI with photodetectors can still be improved by using a second interferometer as a demodulator, resulting however in more complex configurations ${ }^{2}$. On the other hand, the demodulation in the wavelength domain using an optical spectrum analyzer (OSA) has lately gained attention for allowing the use of low-coherence sources such as LED or superluminescent LED (SLED) that reduce intensity fluctuation problems and ambiguity of direction ${ }^{3}$. Designated as a technique of white light interferometric modulation, the wavelength signal can supply absolute values of displacements with sufficient range and resolution ${ }^{4,5,6}$. The use of OSA in the spectrum interrogation provides much higher resolution and sensitivity than the conventional photodetector setup, allowing detection of smaller displacements through the analysis of the spectrum peak power. But due to the OSA slow response time, its use has been limited only to measure of static displacement. However, employing an appropriate demodulation method the measurement of vibration amplitude and resonance frequencies is possible and demonstrated in this work.

\section{EXPERIMENTAL METHODOLOGY}

\subsection{Principle of operation}

The extrinsic Fabry-Perot interferometer was built using the setup shown in Figure 1. A portion of the light coupled into the optical fiber is partially reflected at the cleaved interface of a fiber that, due to contact with air, shows a reflectivity of about $4 \%$. The transmitted intensity is again reflected by the PZT disc which serves as the second mirror. The reflected intensities result in an interference pattern that depends basically on the optical path difference (2d) and the reflectivity of the mirrors. When a light from a low coherence source is reflected by the EFPI and measured by the OSA, the interference spectrum is expressed by ${ }^{3,4}$

$$
I(\lambda)=A(\lambda)+B(\lambda) \cos \left(\frac{4 \pi d}{\lambda}+\varphi\right)
$$

21st International Conference on Optical Fiber Sensors, edited by Wojtek J. Bock, Jacques Albert, Xiaoyi Bao, Proc. of SPIE Vol. 7753, 775319 · (c) 2011 SPIE · CCC code: 0277-786X/11/\$18 · doi: 10.1117/12.885858 
where $A(\lambda)$ is the background introduced by the profile of the light source, $B(\lambda)$ is the contrast that is influenced by the length of the cavity $d$ and $\varphi$ is a constant phase shift introduced by the reflection at the PZT.

An Amonics SLED light source with central wavelength at $1475.7 \mathrm{~nm}$ and $37.6 \mathrm{~nm}$ FWHM was used. The interrogator is an OSA Agilent $86142 \mathrm{~B}$ with a wavelength resolution of $60 \mathrm{pm}$ and sensitivity of $-90 \mathrm{dBm}$. A computer connected to the OSA allowed the control and capture of the optical spectra in the range from 1445 to $1520 \mathrm{~nm}$ with a power of $1 \mathrm{~mW}$. The piezo disc with $2 \mathrm{~mm}$ thickness and $25 \mathrm{~mm}$ in diameter was aligned perpendicular and close to the fiber end face $(\mathrm{d}=20 \mu \mathrm{m})$ which served as the initial reference point. The EFPI calibration was performed with the help of a translational stage. The distance between the fiber and the piezo was varied in $50 \mu \mathrm{m}$ steps up to a maximum distance of $470 \mu \mathrm{m}$, where the fringes visibility had a considerable reduction. For measuring dynamic displacements and the resonant frequence the Tektronix AFG3000 function generator was used to drive the piezo disc with an AC voltage up to $10 \mathrm{~V}$. For each shift caused by the excitation of the disc the spectrum was captured and stored for further processing. Room temperature was controlled in order to avoid any possible influence during the measurement of the fringe pattern.

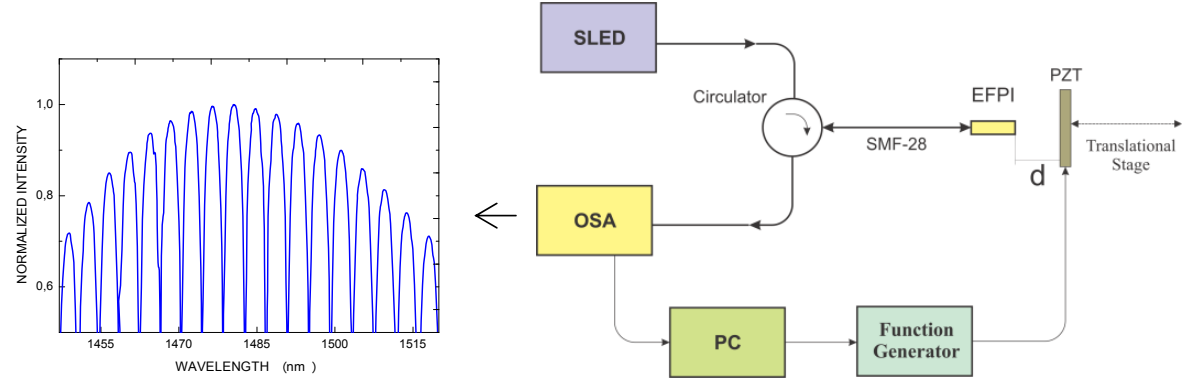

Figure 1. EFPI experimental setup for measuring displacements of the PZT disc.

\subsection{Calibration techniques}

For calibration three signal processing techniques were employed, that are based on the wavelength domain analysis. The first to be investigated was a technique based on the application of the Fast Fourier Transform (FFT) to the spectrum seen in Figure 1. The spectrum is considered as a periodic signal with a frequency $f c$ and modulated in amplitude.

Thus the displacement $d$ of the PZT can be obtained directly by using the FFT of (1) whose carrier frequency corresponds to the peak of $f \mathcal{C}$ sidelobes equally spaced from a central frequency $f$, as given by ${ }^{3,4}$

$$
\begin{gathered}
f_{\mathcal{C}}=\frac{2 d}{\lambda^{2}} \\
I(f)=A(f)+B\left(f-f_{\mathcal{C}}\right)+B\left(f+f_{\mathcal{C}}\right)
\end{gathered}
$$

The second employed method is based on the direct analysis of the spectrum shown in Figure 1. In this method the translational displacements of the piezo disc are obtained by directly monitoring the peaks of two adjacent fringes, as follows ${ }^{2}$

$$
d=\frac{\lambda 1 \lambda 2}{2(\lambda 2-\lambda 1)}
$$

The third demodulation technique is based on the phase shift of the interferometric fringes, and unlike the other two methods it allows the measurement of the distance variation $\Delta d$ related to an initial reference distance $d$, but whose measurement is limited to a dynamic range of $\lambda / 2$. $\Delta d$ can be assessed by monitoring the change in the wavelength, $\Delta \lambda$ of a particular fringe $\lambda$ as given by ${ }^{5}$

$$
\Delta d=\frac{\Delta \lambda}{\lambda} d
$$




\section{RESULTS AND DISCUSSION}

Figure 2a shows the correlation between FFT technique and measurements made manually using the translational stage for the range shown in the inset. The PZT displacements are obtained directly from the peak frequency. The $20 \mu \mathrm{m}$ EFPI resolution shows that below this value only one fringe is present in the cavity. Although the FFT presents low resolution in order to measure the piezo vibration amplitudes, the method showed a good correlation for sensor calibration. The analysis with the second technique, whose results are obtained by monitoring the peaks of two adjacent fringes, showed a better correlation compared to the FFT technique (see inset of Figure 2b). In the third method, the absolute PZT displacements were obtained by combining (4) and (5). Although not demonstrated here, this technique provides a 99,9\% correlation. Figure $2 \mathrm{~b}$ shows the obtained resolution for the second and third techniques. One notes that the $60 \mathrm{~nm}$ resolution of the second technique does not allow one to detect oscillations of the employed piezo disc, which has a piezoelectric charge constant of $5 \mathrm{~nm}$ at $10 \mathrm{~V}$. The third technique, despite its high resolution in the desired working range, was limited by the dynamic displacements presented by the spectral fringes when the PZT was excited.
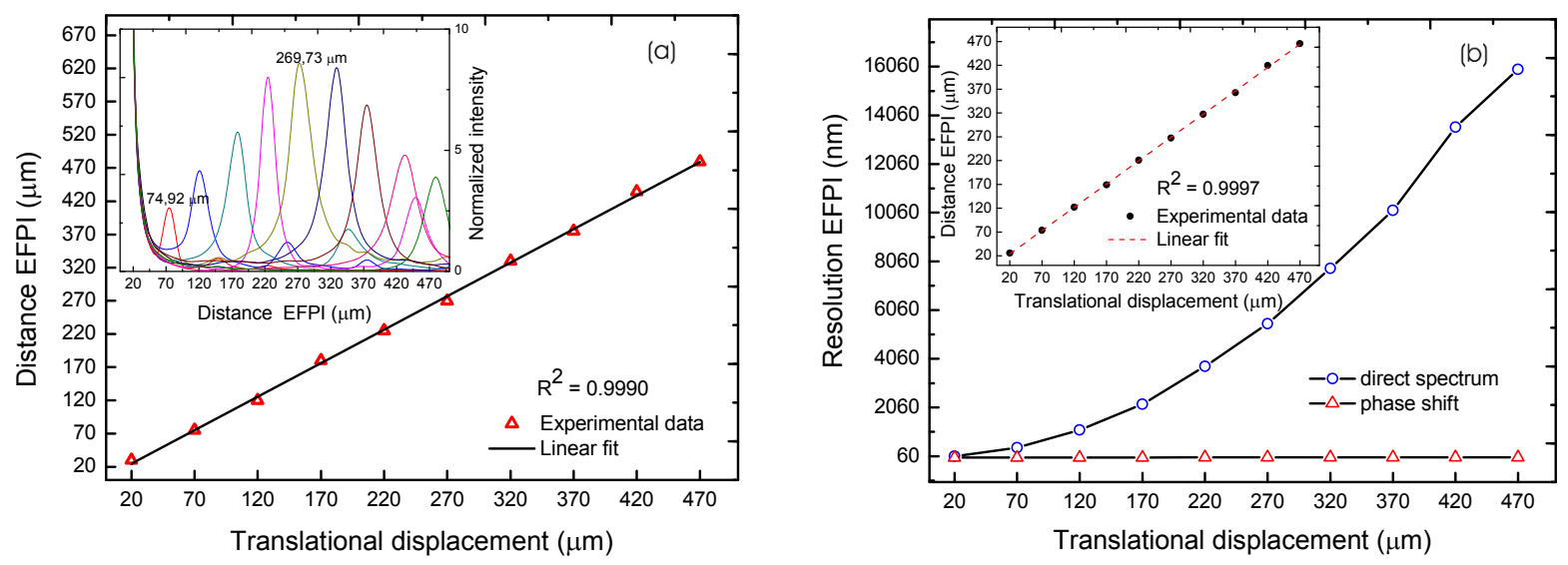

Figure 2. Correlation results using: (a) the FFT, (b) the direct spectrum and the phase shift techniques.

After the sensor calibration has taken place, the EFPI intensity response was obtained. The nonlinear smooth variation of intensity as a function of translational displacement shown in Figure 3a indicates that the EFPI is properly aligned with the piezo disc interface. It is observed that within the displacement range from 20 to $70 \mu \mathrm{m}$, the sensor showed a higher sensitivity. The inset shown in Figure 3a shows an improved characterization of this range with an approximate linear response. For further measurements a reference point corresponding to a displacement of approximately $18 \mu \mathrm{m}$ was chosen. At this point, the PZT was excited with a voltage range from 0 to $10 \mathrm{~V}$ and the variations in wavelength and intensity of the fringe peaks were monitored as seen in the inset of Figure $3 \mathrm{~b}$. The correlation illustrated in Figure $3 \mathrm{~b}$ between the excitation voltage and the dynamic displacement of the PZT shows an approximately linear relationship with a quadratic fit of $97 \%$ and sensitivity of $0.84 \mathrm{~nm} / \mathrm{V}$.
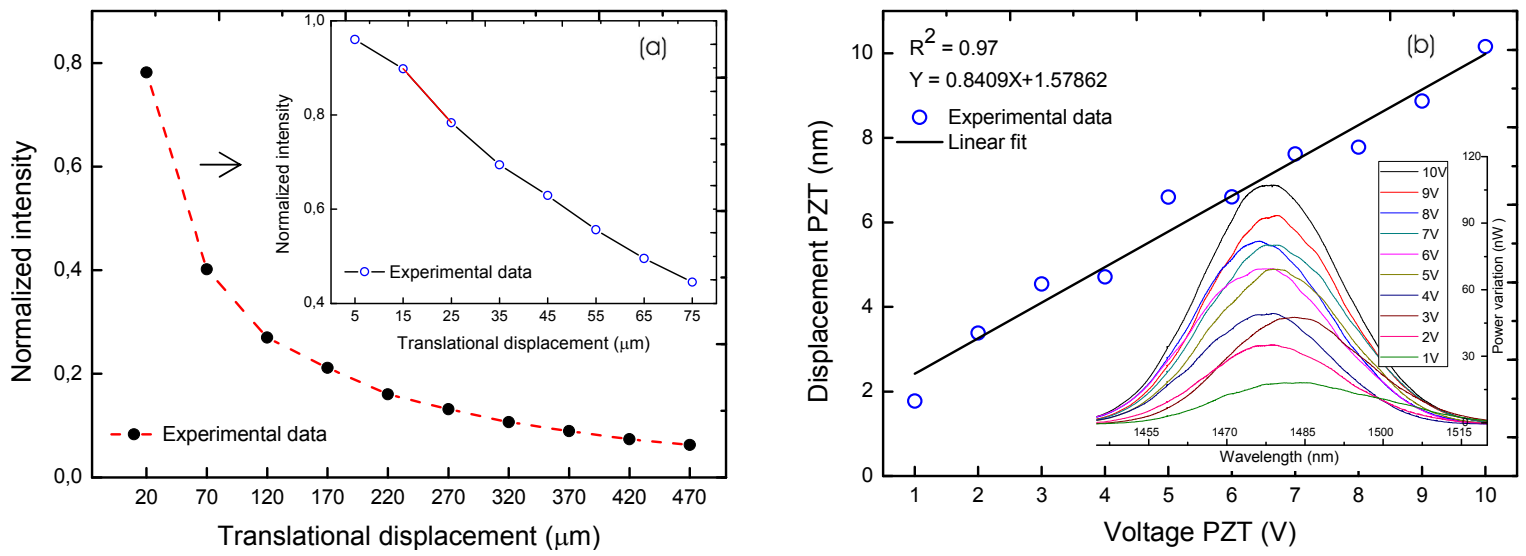

Figure 3. Method for vibration measure: (a) EFPI intensity response, (b) PZT vibration measured by EFPI. 
Using the method described above the PZT resonant frequency was also investigated. In this case, the applied voltage to the disc was kept at constant $10 \mathrm{~V}$ and the frequency of the function generator varied from 50 to $135 \mathrm{kHz}$ in steps of $5 \mathrm{kHz}$. A resonant frequency of approximately $90 \mathrm{kHz}$ was observed with larger oscillation amplitude of the PZT characterized by the maximum intensity reflected in the EFPI (Figure 4a). To check the accuracy of this measure, the PZT resonance was also verified using an Agilent 4294A Precision Impedance Analyzer, which allows verification of the oscillation frequency over the condition of minimum impedance and zero phase. Figure $4 \mathrm{~b}$ shows that the measurement made with the EFPI is consistent with that of the impedance analyzer.
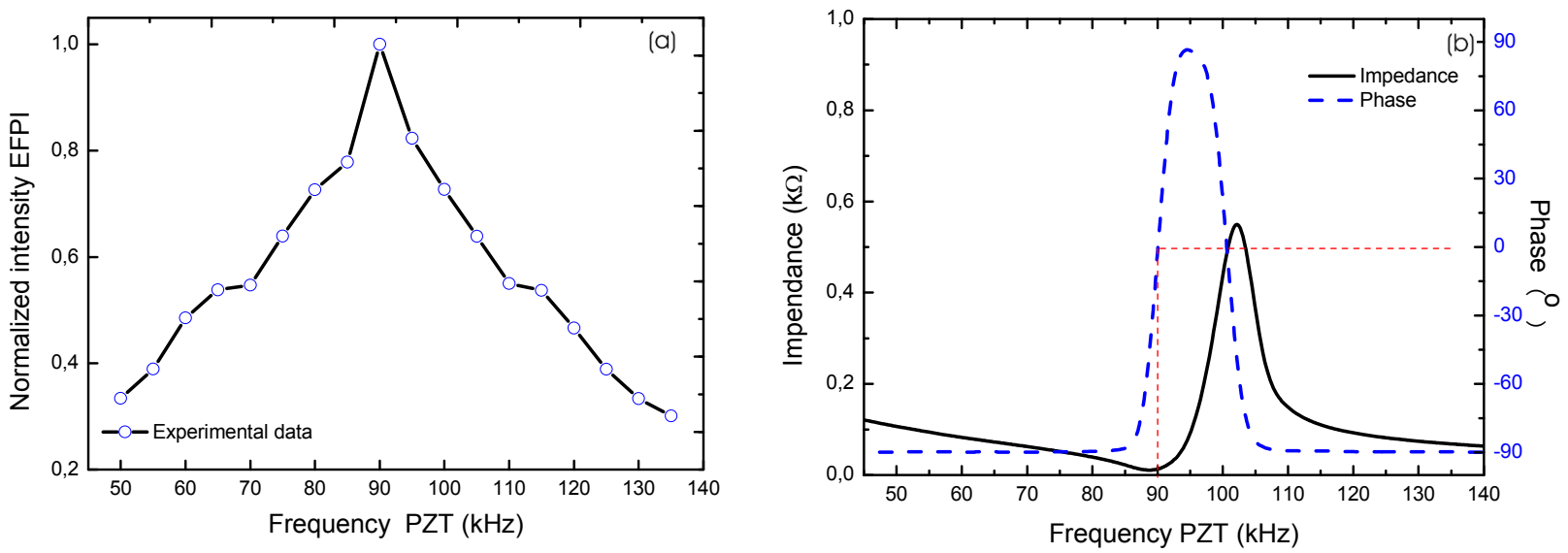

Figure 4. Resonant frequency of PZT: (a) measured by the EFPI (b) measured by the impedance analyzer

\section{CONCLUSION}

A simple method for measuring vibration amplitude and resonances of a piezo disc with a fiber extrinsic Fabry-Perot interferometer and an optical spectrum analyzer has been proposed. The method consists basically in monitoring the peaks of a single interferometric fringe at a point of high sensitivity of the sensor. Thus the vibration amplitude of the PZT can be correlated with variations in intensity, the central wavelength, FWHM or the quality factor of the signal measured by the sensor. The three investigated methods (the demodulation of the Fourier spectrum, the monitoring of adjacent fringes and phase shift) allowed a good calibration of the sensor as compared with static displacements made using a translational stage. Correlations in the order of $99.9 \%$ were achieved. The use of OSA in the verification of the dynamic displacement has the advantage of higher resolution and sensitivity compared to conventional photodetectors. Vibration amplitude characterization with a sensitivity of $0.84 \mathrm{~nm} / \mathrm{V}$ and the measurement of the piezo resonant frequency (at $90 \mathrm{kHz}$ ) shows that the OSA can be used for both static and dynamic displacements resulting in a simple setup that may be employed in various applications.

\section{REFERENCES}

[1] Gangopadhyay, T.K.; Chakravorti, S.; et al "Time-frequency analysis of multiple fringe and nonsinusoidal signals from a fiber-optic vibration sensor using an EFPI," Ligh. Tech. J., 24(5), 2122- 2131(2006)

[2] Jiang, Y. "High-resolution interrogation technique for fiber optic extrinsic Fabry-Perot interferometric extrinsic Fabry-Perot interferometric sensors by the peak-to-peak method" Appl. Opt. 47, 925-932 (2008)

[3] Jiang,Y; "Fourier Transform White-Light Interferometry for the Measurement of Fiber-Optic Extrinsic FabryPerot Interferometric Sensors," Phot. Tech. Letters, 20(2),75-77 (2008)

[4] Shen, F., Wang A., "Frequency-estimation-based signal-processing algorithm for white-light optical fiber Fabry-Perot interferometers," Appl. Opt. 44, 5206-5214 (2005)

[5] Huang, Y. et al "An extrinsic Fabry-Perot interferometer-based large strain sensor with high resolution", Meas. Sci. Technol. 21,105308-105317 (2010)

[6] Majumdar, A., Huang H., "Compact optical fiber white light interferometric distance sensor for arbitrary small distance measurement," Appl. Opt. 48, 3702-3708 (2009) 